\title{
Rapid and Elevated Tumor Responses to Wekley Paclitaxel for AIDS-Related Kaposi Sarcoma
}

\author{
S. Berrad ", K. Oualla, V. W. Lokonga, L. Nouiakh, H. Erraichi, L. Amaadour, Z. Benbrahim, \\ S. Arifi, N. Mellas. \\ Department of Medical Oncology, Hassan II University Hospital, Fez, Morocco \\ *Corresponding Author: Soumia Berrad; soumiaberrad3@gmail.com
}

Received: 13 November 2020;

Accepted: 30 November 2020;

Published: 12 December 2020

\begin{abstract}
Kaposi sarcoma is a rare condition, it is an incurable disease with a poor prognosis, the treatment of this pathology is variable including surgery, Radiotherapy, chemotherapy and antiviral treatments. A number of drugs approved for the treatment of KS associated with AIDS, in particular the pegylated liposomal doxorubicin (DLP) which is currently the standard, Paclitaxel, have also shown efficacy against advanced SK. We report a case of a patient with kaposi's sarcoma associated with HIV metastatic in the lung, liver and lymph nodes and who presented an almost complete response after 4 months of chemotherapy with weekly Paclitaxel at a dose of $100 \mathrm{mg}$ in our department of medical oncology at CHU Hassan II in Fez. Of interest is the complete response to paclitaxel treatment in a patient followed for metastatic kaposi sarcoma.
\end{abstract}

Keyword: Kaposi sarcoma, paclitaxel, VIH, metastasis.

\section{Introduction}

Kaposi (KS) is a neoplasm that originates in the lymphatic endothelium and shows a broad spectrum of clinical manifestations [1]. It is a ubiquitous systemic disease in which the skin manifestations are prominent. Visceral involvement is rare, and remains an indication for treatment with systemic chemotherapy ${ }^{[2]}$.

The treatment options are variable, either surgery, or radiotherapy and chemotherapy associated with antivirals, depending on the stage of the disease, the mode of evolution, the clinic, and the status of the immune system, the main objective of treatment is to improve the quality of life.

We report a case of a patient with an almost complete response after 6 months of weekly treatment with Paclitaxel with multiple visceral locations of Kaposi's sarcoma associated with HIV.

\section{Observation}

This is a 35-year-old patient who has been followed in the medical oncology department at the Hassan II university center since 2013 for chronic HIV infection who has been on antiretroviral treatment with zidovudine, lamivudine, efavirenz with regular monitoring.

After 10 months of treatment, she revealed brownish spots on the body, associated with dysphagia, dysphonia and hearing loss, progressing in a context of unstated weight loss and fever.
The objective clinical examination of the multiple purplish angiomatous lesions, scattered over the four limbs of varying sizes taking the thorax and face associated with tonsillar hypertrophy and invading the tongue.

The tonsillar biopsy with a histological study coming back in favor of kaposi's sarcoma.

The extension workup consists of an objective thoracoabdomino-pelvic CT scan of sub-diaphragmatic and peripheral lymph node metastases with hepatic pulmonary involvement.

Ideally, the patient would be put on a systemic chemotherapy regimen including bleomycin, however, plethysmography revealed a restrictive pulmonary syndrome contraindicated for bleomycin. The patient was put under paclitaxel at a dose of $100 \mathrm{mg}$ hebdomendary with haematological complication persistent neutropenia with treatment interruption for 3 weeks, at the end of the aplasia we resumed treatment, the patient was evaluated after four months, and clinically we noted a significant decrease in tonsillar lesions and on computed tomography the disappearance of pulmonary and hepatic lesions. We continued the treatment for a total of 4 months with good clinical and biological tolerance. She is currently in complete remission after regular follow-up for 8 years. 


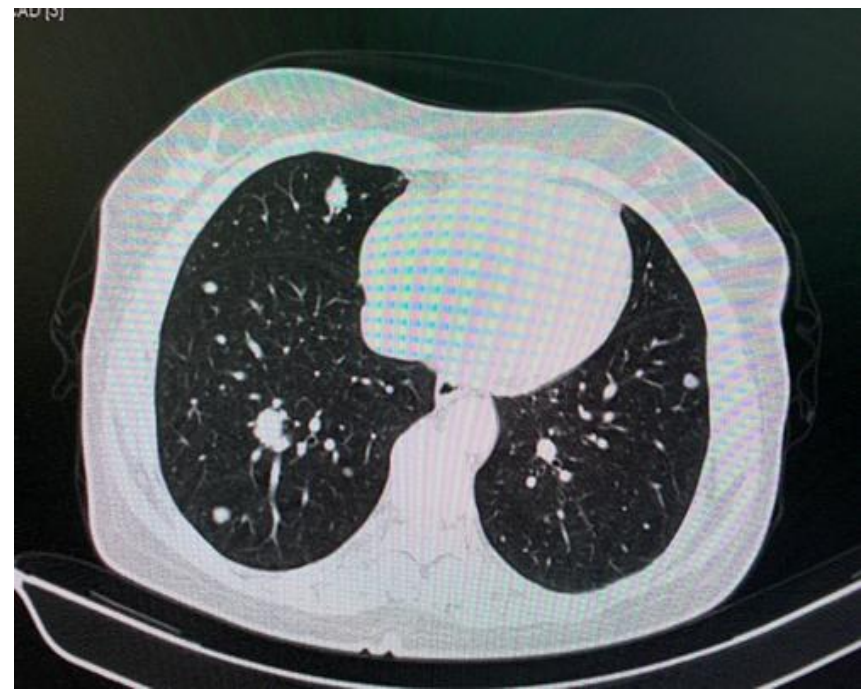

Figure 1: CT of chest revealed multiple pulmonary metastasis upon diagnosis.

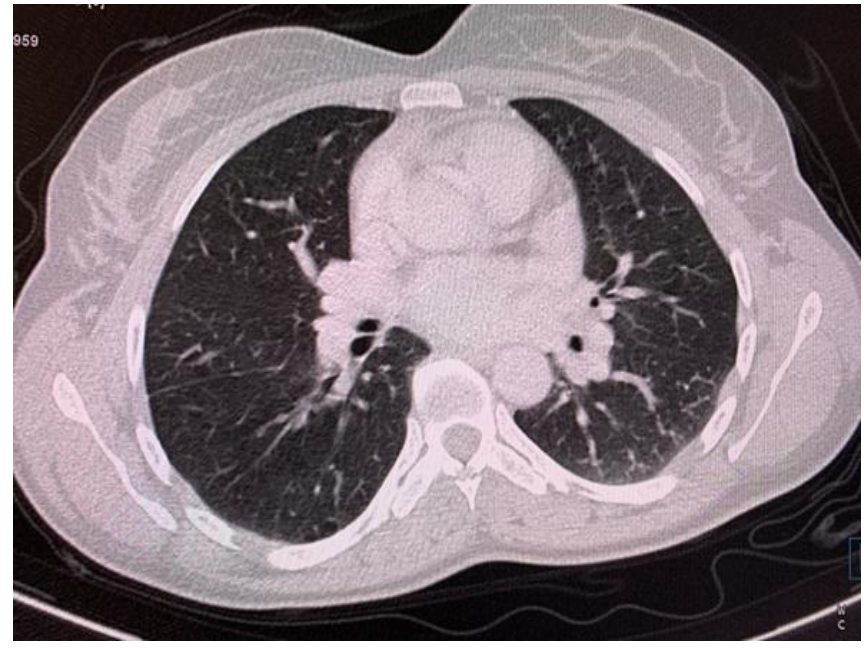

Figure 2: CT chest showing the disappearance of pulmonary lesions after treatment with chemotherapy paclitaxel.

\section{Discussion}

There are four forms of Kaposi's sarcoma, the classic form mainly affects elderly men, the African form was recognized in young men and children in Central Africa, iatrogenic SK mainly in transplant patients but also due to chemotherapy and to immunosuppressive treatments ${ }^{[3]}$, the epidemic form associated with HIV has been described in homosexual men ${ }^{[4]}$, our patient presents the latter form. These forms are distinguished by a different geographical distribution and clinical presentation, but they have in common the same type histological despite their extremely variable clinical pictures.

AIDS-related KS is a multifocal proliferative disease of skin and visceral expression. Clinically, it can appear as nodules or pink purple spots on the skin or mucous membranes ${ }^{[5]}$.

The course of this disease varies from one patient to another. ART treatment has considerably reduced the incidence of KS associated with AIDS as well as the morbidity and mortality of this pathology ${ }^{[6]}$. Local treatment modalities such as intra-lesional vinblastine, cryotherapy is reserved for patients with limited skin disease without systemic dissemination ${ }^{[8]}$.

However, visceral involvement is an indication for systemic treatment. Several drugs are active in AIDS-related kaposi sarcoma include lyposomal doxorubicin which is first-line FDA approved according to a randomized controlled clinical trial that demonstrated pegylated liposomal doxorubicin is superior to the $\mathrm{ABV}$ combination, with response rates of $45.9 \%$ for pegylated liposomal doxorubicin versus $24.8 \%$ for the $\mathrm{ABV}$ combination ${ }^{[7]}$. Paclitaxel has also shown efficacy with moderate toxicity in patients with advanced AIDS-related KS.

In a 2008 study evaluating the efficacy and safety of paclitaxel at a dose of $100 \mathrm{mg}$ per week in 17 patients with advanced and refractory KS 14 patients showed a complete and / or partial response ${ }^{[9]}$.

A randomized trial comparing the efficacy and toxicity of paclitaxel and doxorubicine liposomal pegylée was carried out in 2010 by Cianfrocca $M$ et al.This study included 73 patients in two arms. This comparison revealed comparable response rates $(56 \%$ vs $46 \%$; $\mathrm{P}=0.49$ ), median progression-free survival (17.5 months vs. 12.2 months; $\mathrm{P}=0.66)^{[10]}$.

Our patient received paclitaxel due to the unavailability of pegylated liposomal doxorubicin, treatment was administered with low dose paclitaxel to minimize toxicity. A major response and good tolerance were observed, suggesting that weekly paclitaxel may be effective as a first-line chemotherapy in the treatment of AIDS-related KS with visceral involvement.

\section{Conclusion}

AIDS-related Kaposi's sarcoma is an incurable disease with a poor prognosis in the presence of visceral metastases at least weekly paclitaxel is a better first-line therapeutic option than the more toxic combination chemotherapy, as long as pegylated doxorubicine liposomal which is currently unavailable and not affordable in our country.

\section{Ethics approval and consent to participate}

Our manuscripts do not report studies involving human or animal participants.

\section{List of abbreviations}

DLP: Pegylated Liposomal Doxorubicin

KS: Kaposi Sarcoma

ABV: Doxorubicin Bleomycin Vincristin

\section{Conflicts of Interest}

The authors do not disclose a conflict of interest regarding the publication of this article.

\section{Funding Statement}

The article is not financed.

\section{Authors' contributions}

All authors read and approved the final manuscript.

\section{References}

[1] Kaposi's sarcoma of the lymphangioma type: report of four cases and review.

Mohanna S, Sanchez J, Ferrufino JC, Bravo F, Gotuzzo EJ Eur Acad Dermatol Venereol. 2006 Sep; 20 (8): 10101. [PubMed] [List of references]. 
[2] Kaposi M. Idiopatisches multiple pigmentosarcom der Haut. Arch Dermatol Syphil 1872; 4: 265-73.

[3] Dupin N. Kaposi's disease Rev Med Int 1995; 16: 484486 [cross-ref].

[4] Lymphangiectatic Kaposi's sarcoma in an AIDS patient.Santos M, Vilasboas V, Mendes L, Talhari C, Talhari S. Un Bras Dermatol. 2013 March-April; 88 (2): 276-8. doi: 10.1590 / S0365-05962013000200019.PMID: 23739700 Free PMC article.

[5] Kaposi's sarcoma K Antman 1, Y ChangPMID: 10749966DOI: 10.1056 / NEJM200004063421407.

[6] Kaposi's SarcomaKaren Antman, M.D., and Yuan Chang, M.D.

Kaposi's sarcoma: an update. Schwartz RA, J Surg Oncol. September 1, 2004; 87 (3): 146-51. [PubMed] [List of references].

[7] Northfelt DW, Dezube BJ, Thommes JA, Miller BJ, Fischl MA, Friedman-Kien A, et al. Pegylated liposomal doxorubicin versus doxorubicin, bleomycin, and vincristine in the treatment of AIDS-related Kaposi's sarcoma: results from a randomized phase III clinical trial. J Clin Oncol 1998; 16: 2445-51. [PUBMED] [FULLTEXT].

[8] Kaposi's sarcoma in an HIV-positive person successfully treated with paclitaxelAtul Dongre, Chiara MontaldoMedicines sans Frontieres Belgium (MSF B). HIV / AIDS Khar Clinic, Mumbai, India.

[9] Brambilla L, Romanelli A, Bellinvia M, Ferrucci S, Vinci M, Boneschi V, et al. Weekly paclitaxel for advanced aggressive classic Kaposi sarcoma: Experience in 17 cases. $\mathrm{Br} \mathrm{J}$ Dermatol 2008; 158: 1339-44. [PUBMED] [FULLTEXT].

[10] Cianfrocca M, Lee S, Von Roenn J, et al. Randomized trial of paclitaxel versus pegylated liposomal doxorubicin for advanced human immunodeficiency virus-associated Kaposi sarcoma: evidence of symptom palliation from chemotherapy. Cancer. 2010; 116 (16): 3969-3977. doi: 10.1002 / cncr.25362. 